\title{
Las enzimas involucradas en el metabolismo de la cocaína: Una nueva aproximación farmacológica para el tratamiento de la intoxicación por sobredosis de cocaína
}

\author{
Alberto Salazar-Juárez, ' Susana Barbosa Méndez,' Noé Jurado, 'Benito Antón ${ }^{1 \dagger}$
}

\section{Artículo de revisión}

\section{ABSTRACT}

\section{Introduction}

New therapeutic strategies against cocaine overdose toxicity have been developed. These new approaches are based on the design and synthesis of proteins involved in the destruction of cocaine before it has a chance to penetrate nerve tissue.

\section{Objective}

To review the progress in the effect of the increase in the catalytic activity of BChE and hCE enzymes produced for the treatment of patients in cocaine overdose toxicity conditions in order to determine the advantages and disadvantages of its use. Its potential future use in patients channeled by a cocaine overdose is also explored.

\section{Method}

A bibliographic search was conducted using PubMed; descriptors were "cocaine", "hydrolase", "esterase" and "butyrylcholinesterase". 220 papers were obtained and 126 papers were used for these review.

\section{Results}

The $\mathrm{BChE}, \mathrm{COCH}$ and Coce bacterial enzymes significantly decrease the levels of cocaine in blood and brain and thereby attenuate the effects of a cocaine overdose.

\section{Discussion and conclusion}

The results obtained in animal models suggest the potential therapeutic use of these enzymes in humans to rapidly inactivate cocaine and develop treatments to stop deaths associated with cocaine overdose intoxication. These enzymatic approaches offer a novel therapeutic application to treat cocaine overdose.

Key words: Addiction, cocaine, enzymes and pharmacotherapy.

\section{RESUMEN}

\section{Introducción}

Se han desarrollado nuevas estrategias terapéuticas contra la toxicidad por sobredosis de cocaína basadas en el aumento en la actividad catalítica de enzimas que participan en la destrucción de su molécula, antes de que tenga la oportunidad de penetrar el tejido nervioso.

\section{Objetivo}

Describir los avances en el efecto del aumento en la actividad catalítica de las enzimas $B C h E$ y las $h C E$, producidas para el tratamiento de pacientes en condiciones de toxicidad por sobredosis de cocaína, así como mencionar sus ventajas y desventajas y su potencial uso futuro en pacientes internados por una sobredosis de cocaína.

\section{Método}

Se realizó una búsqueda bibliográfica por medio del PubMed, usando como descriptores las palabras "Cocaine", "hydrolase", "esterase" y "butyrylcholinesterase". Se obtuvieron 220 artículos de los cuales se usaron 126 para esta revisión.

\section{Resultados}

Las enzimas $\mathrm{BChE}, \mathrm{COCH}$ y $\mathrm{CoCe}$ bacteriana disminuyeron significativamente los niveles de cocaína en la sangre y el cerebro y con ello atenuaron los efectos de una sobredosis de cocaína.

\section{Discusión y conclusión}

Los resultados obtenidos en modelos animales sugieren el potencial terapéutico del uso de estas enzimas en humanos, para inactivar rápidamente a la cocaína y desarrollar tratamientos para evitar las muertes asociadas con la intoxicación por sobredosis.

Estas metodologías enzimáticas ofrecen una aplicación terapéutica novedosa para el tratamiento de la sobredosis.

Palabras clave: Adicciones, cocaína, enzimas, terapia farmacológica.

Subdireccion de Investigaciones Clínicas. Instituto Nacional de Psiquiatría Ramón de la Fuente Muñiz.

Correspondencia: Dr. Alberto Salazar-Juárez. Laboratorio de Neurobiología Molecular y Neuroquímica de las Adicciones, Subdirección de Investigaciones Clínicas, Instituto Nacional de Psiquiatría Ramón de la Fuente Muñiz. Calz. México-Xochimilco 101, San Lorenzo Huipulco, Tlalpan, 14370, Cd. de México. Tel: +52 554160 - 5094. E-mail: azazel_vamp@yahoo.com.mx

Recibido primera versión: 22 de diciembre de 2015. Segunda versión: 19 de octubre de 2016. Aceptado: 24 de octubre de 2016. 


\section{INTRODUCCIÓN}

La farmacopea clásica empleada para atenuar y/o abolir la dependencia a drogas de abuso ilegal, con alta potencia adictiva como la cocaína, ha demostrado una eficacia terapéutica limitada, tanto al corto como al largo plazo. ${ }^{1,2} \mathrm{Ba}-$ sado en este desalentador panorama clínico-terapéutico, desde hace más de una década diversos investigadores han desarrollado nuevas estrategias terapéuticas contra la adicción a drogas como la cocaína.,

Algunos grupos de investigación han desarrollado terapias farmacológicas mediante el uso de nuevos fármacos,, 6 otros han validado métodos de inmunoterapia basados en procedimientos de vacunación activa y pasiva ${ }^{7,8}$ y unos más han explorado el uso de proteínas que involucran la destrucción de la molécula de cocaína, antes de que tenga la oportunidad de cruzar la barrera hemato-encefálica y penetrar en el tejido nervioso (figura 1-B), como es el aumento en la actividad catalítica de enzimas como la butiril-colinesterasa (BChE) $)^{9-11}$ y la carboxilo-esterasas hepáticas (hCE-1 y hCE-2).

Diversos estudios epidemiológicos han reportado que un gran porcentaje de las muertes relacionadas al abuso de cocaína están generalmente relacionadas a una intoxicación por una sobredosis, debido principalmente a que se carece de una terapia eficaz. ${ }^{12}$ Desde hace varios años diversos
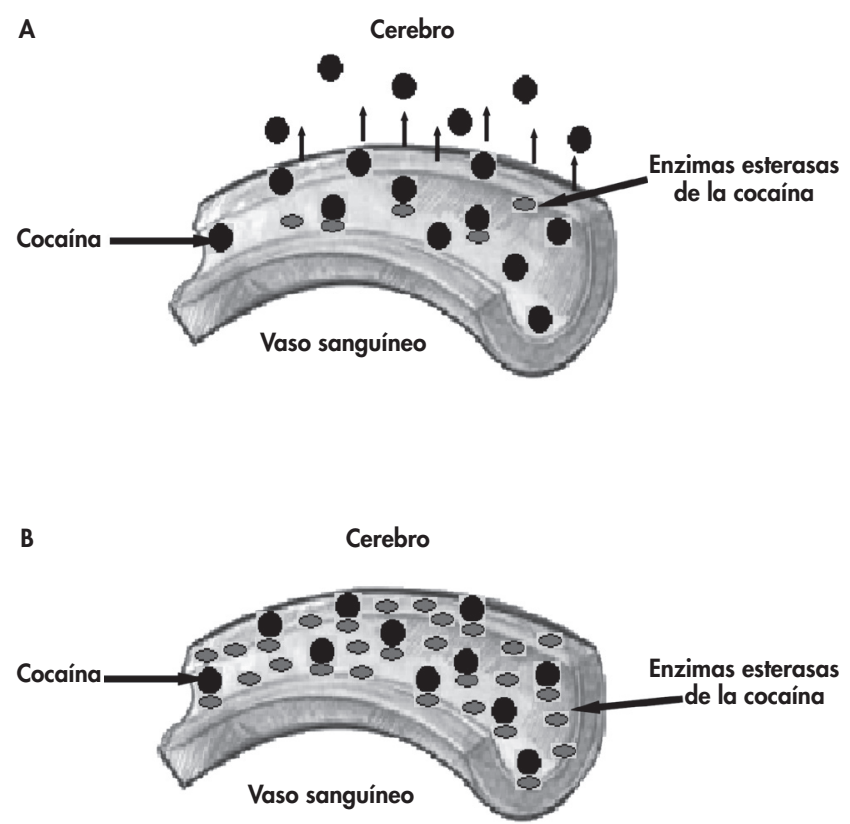

Figura 1. (A) La cocaína rápidamente se localiza dentro de los vasos sanguíneos, cruza la barrera hemato-encefálica y alcanza a su sitio blanco dentro del cerebro. Dentro de los vasos sanguíneos se localizan enzimas que hidrolizan a la cocaína (BChE, CocE), pero son pocas y su capacidad hidrolítica es limitada, por lo que son poco eficientes. (B) Cuando se administran enzimas puras o modificadas genéticamente (incrementa su capacidad de hidrólisis y su vida media) capturan rápidamente a la cocaína dentro del vaso, la hidrolizan en sus metabólitos inactivos e impiden que se lleven a cabo sus efectos reforzantes o tóxicos. grupos de investigación han realizado estudios encaminados a desarrollar y validar algunas estrategias terapéuticas con relativo éxito. Como se mencionó anteriormente, una de estas estrategias ha sido el aumento en la actividad catalítica de las enzimas encargadas de metabolizar a la molécula de la cocaína. Se han reportado diversos estudios describiendo cómo, mediante técnicas de biología molecular, se ha logrado potenciar la actividad de esta(s) enzima(s); otros estudios han descrito el efecto del tratamiento con estas enzimas en roedores y en humanos. Sin embargo, se carece de una revisión que describa los beneficios, ventajas, desventajas y el potencial uso futuro del aumento de la actividad catalítica de las enzimas encargadas de metabolizar a la cocaína, la BChE y las hC. El objetivo de esta revisión fue analizar los avances científicos relacionados con el aumento en la actividad catalítica de las enzimas BChE y las hCE, con el fin de describir sus principales efectos biológicos y su posible uso para el tratamiento de pacientes en condiciones de toxicidad por sobredosis de cocaína.

\section{MÉTODO}

La búsqueda bibliográfica se realizó usando el motor de búsqueda "Entrez PubMed"; en la búsqueda se utilizaron las siguientes palabras clave: Cocaine, butyrylcholinesterase, hydrolase y esterase. La búsqueda se realizó abarcando un periodo de enero de 1970 a diciembre de 2015. El algoritmo de búsqueda fue: ("cocaine"[MeSH Terms] OR "cocaine"[All Fields]) AND ("hydrolases"[MeSH Terms] OR "hydrolases"[All Fields] OR "hydrolase"[All Fields]) AND ("esterases"[MeSH Terms] OR "esterases"[All Fields] OR "esterase"[All Fields]) AND ("cholinesterases"[MeSH Terms] OR "cholinesterases"[All Fields] OR "butyrylcholinesterase"[All Fields] OR "butyrylcholinesterase"[MeSH Terms]).

Los criterios de inclusión fueron: 1) Estudios publicados en revistas internacionales indexadas, 2) artículos de investigación básica, preclínica, clínica y de revisión que 3) describan la estructura, bioquímica y cinética de las enzimas BChE y hCE, así como la caracterización del efecto biológico-terapéutico y de seguridad biológica, 4) en animales (roedores, conejos y primates) y humanos adultos, 5) estudios que se realizaron en Estados Unidos, Canadá y la Comunidad Europea y 6) que fueron publicados en idiomas inglés, francés y español.

Los criterios de exclusión fueron: que los artículos fueran 1) editoriales, opiniones de expertos o comunicaciones a congresos, 2) que los artículos, en su contenido, no incluyeran información acerca del objetivo del estudio, 3) su contenido se encontrara repetido en el contenido de otro artículo.

El análisis de los resultados indicó que la búsqueda bibliográfica arrojó un total de 220 artículos, de los cuales sólo 126 fueron considerados para la inclusión en esta revisión. 
De estos 126 artículos, 97 son artículos de investigación, nueve de investigación clínica, un meta-análisis y 19 artículos de revisión (figura 2).

\section{RESULTADOS}

\section{Butiril-colinesterasa}

La cocaína, una vez ingerida, es metabolizada casi en su totalidad. La principal vía de transformación es la hidrólisis enzimática, siendo las esterasas plasmáticas (BChE) y hepáticas (hCE-1) las principales enzimas responsables de la formación de sus metabólitos: éster de metilo-ecgonina, ecgonina y benzoil-ecgonina (figura 1-A).

La BChE es la principal enzima que metaboliza a la cocaína en el plasma, tanto en humanos como en otras especies. $^{13-16}$

La vida media de la BChE en el plasma de los animales es de aproximadamente 21.6 horas ${ }^{17-19}$ y metaboliza rápidamente a la molécula de cocaína ${ }^{20-23}$ en el metabolito éster de metilo-ecgonina; en cambio, las enzimas hepáticas transforman a la cocaína en los metabólitos norcocaina y benzoílo-ecgonina. ${ }^{20,24-28}$ Este cambio en el perfil metabólico de la cocaína tiene importantes implicaciones fisiológicas. Algunos estudios han demostrado que la benzoil-ecgonina es un potente vaso-constrictor ${ }^{29,30}$ y genera crisis convulsivas; ${ }^{31}$ por su lado, la norcocaína es un metabolito muy hepatotó- xico y un poderoso anestésico local. ${ }^{32,33}$ En cambio, el éster de metilo-ecgonina no genera ningún efecto fisiológico adverso y es rápidamente eliminado por el riñón, por lo que el incremento en la concentración de este metabolito no genera efectos tóxicos en el sujeto. ${ }^{34}$

Diversa evidencia clínica sugiere que la actividad de la BChE endógena está inversamente correlacionada con la severidad de la toxicidad que la cocaína puede generar en humanos. ${ }^{35,36}$ Los niveles normales de BChE varían entre los individuos y son dependientes de la edad, el estado de salud, la exposición a toxinas ambientales y a factores genéticos. ${ }^{37-39}$

Algunos reportes clínicos indican que los individuos que sufren de graves problemas médicos después del uso de la cocaína, tienden a mostrar una menor actividad en la BChE plasmática que aquellos que experimentan problemas menos severos. ${ }^{40-42}$ Adicionalmente, algunos estudios genéticos han reportado que en casos extremos de intoxicación con cocaína, los pacientes homocigotos muestran, o bien una variante "silente" de la BChE, la cual no expresa actividad catalítica detectable ${ }^{43,44} \mathrm{o}$ bien niveles bajos de expresión de la $\mathrm{BChE}$, o incluso muestran variantes defectuosas o "atípicas" de la enzima. Estos pacientes experimentan respuestas prolongadas a la cocaína. Estudios in vitro demostraron que la BChe proveniente de sueros de pacientes atípicos, muestran una disminución del $50 \%$ en la capacidad para hidrolizar a la cocaína en el plasma, ${ }^{45,46}$ lo cual sustenta la importante labor que lleva a cabo la BChE en el suero de sujetos dependientes a la droga.
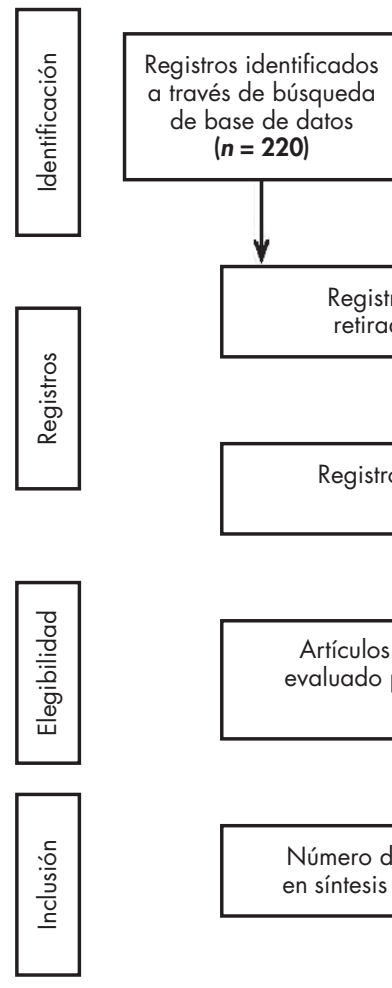

Registros identificados a través de otras fuentes $(n=0)$

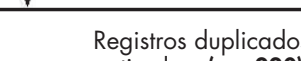

retirados $(\boldsymbol{n}=\mathbf{2 2 0})$
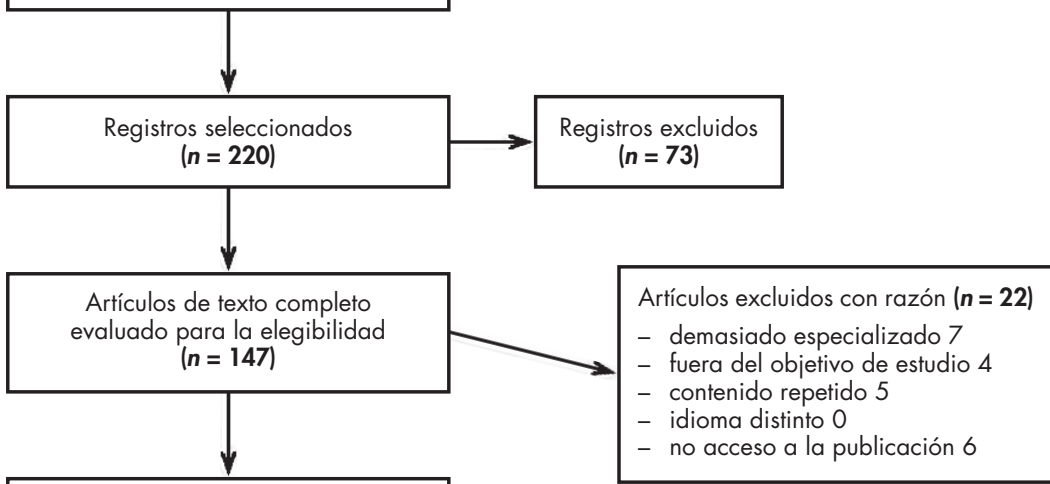

Número de estudios incluidos

en síntesis cualitativa $(n=125)$

Figura 2. Diagrama de flujo del proceso de selección del estudio. 
Algunos estudios pioneros han reportado que pacientes dependientes a la cocaína que han recibido BChE humana purificada (obtenida de suero de donantes), no han presentado eventos clínicos adversos hasta por dos días, ${ }^{47,48}$ lo cual sugiere que la administración de BChE podría ser una terapia útil para tratar a pacientes dependientes a la cocaína.

En modelos animales, la administración diaria por siete días de cocaína (20 mg/kg ip.), a ratones knockout de BChE, el cual expresa una baja o nula actividad para catalizarla, generó rápidamente cardiomiopatías, depresión respiratoria (aproximadamente por 12 horas), patrones respiratorios anormales (apneusis) y, a nivel histológico, hepatotoxicidad significativa y fibrosis peri-vascular cardiaca. ${ }^{48}$ En cambio, ratones con una expresión de $\mathrm{BChE}$ normal recuperaron el ritmo respiratorio a valores normales 30 minutos después de la dosificación y no mostraron apneusis ni hepatotoxicidad. ${ }^{49-51}$

Recientemente se reportó el desarrollo de un ratón mutante-doble, el cual mostraba una expresión nula de la carboxyl-esterasa y de la BChE. Cuando se administró una dosis letal de cocaína $(100 \mathrm{mg} / \mathrm{kg})$, los ratones doble knockout mostraron un incremento ( 2.5 veces) en la duración de los signos tóxicos (hipotermia, hiperactividad, conductas estereotipadas, efectos oculares y flexión dorsal de la cola) en comparación con la duración mostrada por el ratón $\mathrm{BChE}$ naive. ${ }^{50}$

Diversas evaluaciones han reportado que la administración de BChE (15 000 o 5000 IU, iv.), derivada de suero de caballo, disminuyó la vida media de la cocaína de 26.2 minutos a 16.4 minutos en el plasma de roedores, gatos y primates. ${ }^{26,52,53}$ Además, in vitro, la BChE de roedores, primates y humanos, también incrementó el metabolismo de la cocaína. ${ }^{54-56}$

Con respecto a los niveles de cocaína en el cerebro, la administración de BChE (7.8 mg/ kg, iv.) a ratas, redujo la concentración de cocaína en un $80 \%$ a los cuatro minutos, un $30 \%$ a los 45 minutos y un $25 \%$ a los 52 minutos posteriores a la administración de cocaína $\left(30 \mathrm{mg} / \mathrm{kg}\right.$, ip.). ${ }^{26,57-59}$

En ratas se ha reportado que la administración intravenosa de 5000 IU, de BChE derivada de suero de caballo seguido de la administración intraperitoneal de $17 \mathrm{mg} / \mathrm{kg}$ de cocaína produjo una atenuación significativa de la actividad locomotora inducida por su administración, en sesiones de 120 minutos. ${ }^{56,60}$ También disminuyó de manera temporal el restablecimiento de su auto-administración. ${ }^{61-64}$

En roedores y primates la toxicidad aguda inducida por una sobredosis de cocaína estuvo marcada por un incremento en la presión sanguínea, una disminución en el ritmo cardíaco, hipertensión, bradicardia, supresión respiratoria y convulsiones tónico-clónicas, estas últimas están asociadas con crisis epilépticas y son los mecanismos primarios, responsables de la letalidad inducida por la sobredosis de cocaína. ${ }^{65-67}$

La administración de una dosis de BChE de $7.8 \mathrm{mg} /$ $\mathrm{kg}$, iv. incrementó los niveles plasmáticos de la enzima a más de 800 veces de lo normal, lo cual evitó la hipertensión y las arritmias cardiacas inducidas por una sobredosis de cocaína, en ratas (Lynch 1997). Dosis más altas (13.7 o 27.4 $\mathrm{mg} / \mathrm{kg}$ ) disminuyeron la incidencia de crisis convulsivas y muerte producidas por dosis de hasta $80 \mathrm{mg} / \mathrm{kg}$, ip., en el ratón. ${ }^{68}$

Sin embargo, a pesar de su disposición estratégica en la circulación, la eficiencia catalítica de la BChE humana es muy baja y depende de muchos factores. En situaciones de exposición aguda a concentraciones toxicas de cocaína, la BChE es fácilmente superada. ${ }^{69,70}$

Con el objetivo de aumentar la capacidad catalítica de la BChE humana, diversos grupos de investigación realizaron mutaciones sucesivas a la hBChE. ${ }^{71-73} \mathrm{Al}$ introducir una simple mutación, alanina 328-tirosina, en células transfectadas de ovario de hámster, algunos grupos de investigación lograron incrementar la velocidad de hidrólisis de la cocaína por un factor de $4{ }^{74}$ en cambio, si la mutación era una tirosina 332-alanina, la velocidad de reacción aumentaba 40 veces. En ratas, la administración de la BChE mutante bloqueó las crisis convulsivas y la letalidad inducida por una sobredosis de cocaína $\left(100 \mathrm{mg} / \mathrm{kg}\right.$, ip) ${ }^{75}$

\section{Hidrolasa de la cocaína}

Posteriormente, mediante estudios de diseño molecular por computadora y de ingeniería genética, ${ }^{76-80}$ se generaron diversas enzimas capaces de hidrolizar a la cocaína a partir de la BChE humana, a las que se les denominó hidrolasas de la cocaína (hCocE). Después se diseñó un doble mutante llamado "hCocH", un mutante cuádruple denominado "AME$359^{\prime \prime},{ }^{81,82}$ y recientemente se reportó una hBChE con cinco mutaciones simultáneas: "hCocH2". 83

In vitro, la hidrolasa "hCocE" (A328W/Y332A-BChE) tuvo la capacidad de aumentar hasta por 1500 veces la eficiencia catalítica mostrada por la BChE. ${ }^{84-86}$ Sin embargo, a pesar del incremento en la eficiencia en la hidrólisis de la cocaína, la enzima no fue capaz de hidrolizar a la acetilcolina.

Cuando se administró la hCocE ( $3 \mathrm{mg} / \mathrm{kg}$ iv.) a ratas, ésta fue capaz de remover rápidamente la cocaína de los vasos sanguíneos, reduciendo la vida media de la cocaína de 52 a 18 minutos, disminuyó la concentración de cocaína en el plasma, reduciendo así su acumulación en el SNC, e incrementó los niveles plasmáticos de ácido benzoico, un producto no tóxico de la hidrólisis de la cocaína. ${ }^{87,88}$

In vivo, la hCocE disminuyó la actividad locomotora $\mathrm{y}$ atenuó la respuesta cardiovascular (presión sanguínea) inducida por la droga. ${ }^{89-93}$

En estudios de sobredosis de cocaína, en ratas, la hCocE ha demostrado una eficiencia catalítica y selectividad superior, comparada con la hBChE. La hCocE bloqueó eficientemente los efectos cardiovasculares y neurológicos inducidos por dosis letales (180 mg/kg ip.) en rata y primate. ${ }^{65}$ Así, 1 $\mathrm{mg} / \mathrm{kg}$ de hCocE protegió al 100\% de los animales que recibieron una dosis tóxica de cocaína $(180 \mathrm{mg} / \mathrm{kg})$, en cambio, 
la administración de 13 mg de BChE falló en proteger a ratas de la letalidad inducida por una dosis similar. Además, la hCocE dada a ratas después de la aparición de las crisis convulsivas no sólo acortó la duración de éstas sino que también salvó al sujeto de la muerte. ${ }^{94}$

Sin embargo, a pesar de estos resultados, una gran desventaja de la hCocE es que tiene una vida media muy corta (<10 minutos) en el plasma, lo cual no le permite ejercer su protección a largo plazo.

\section{Esterasa bacteriana de la cocaína}

La bacteria rhodococcus sp., MB1, es capaz de producir una esterasa, la bCocE, capaz de hidrolizar a la cocaína tanto in vitro como in vivo. ${ }^{95}$ La actividad enzimática de esta esterasa se logró incrementar hasta x1000 veces más en comparación con la mostrada por la hBChE humana, 105-106 veces más rápida que un anticuerpo monoclonal. ${ }^{96}$

La administración de bCocE atenuó el restablecimiento de la conducta de búsqueda de la droga en animales entrenados previamente a auto-administrarse la cocaína y bloqueó el incremento en la actividad locomotora, inducido por ella. ${ }^{97}$

Además, la bCocE, en dosis de $28 \mathrm{mg} / \mathrm{kg}$, restauró rápidamente (tres minutos) la presión sanguínea, disminuyó la hipertensión, la arritmia cardiaca y redujo la toxicidad inducida por una sobredosis (100 mg/ kg, ip.), previniendo la muerte por crisis convulsivas, en ratas y en el ratón. ${ }^{98}$

Sin embargo, a pesar de su eficiencia las enzimas de mamíferos son más estables in vivo que las bacterianas. La esterasa bacteriana de cocaína inyectada a ratas tuvo una vida media de sólo 15 minutos, comparada con ocho horas para la CocH-albúmina humana. ${ }^{66}$

Los factores que intervienen en la duración de la vida media de la bCocE son múltiples, pero los más relevantes son la respuesta inmune generada por el huésped en contra de la enzima y la temperatura. Brim et al. reportaron que el proceso de eliminación de la bCocE bacteriana fue dependiente de la temperatura (termolábil). La bCocE tiene una vida media de sólo 11 minutos a $37^{\circ} \mathrm{C} .{ }^{99}$

Por su lado, Ko et al. demostraron que a pesar de que la bCocE es una proteína de bacterias muy grande, por lo que es probable que pueda generar una respuesta inmune potente, la bCocE retiene su efectividad después de una o múltiples exposiciones, lo cual sugiere que la CocE es un antígeno débil y no es capaz de generar una respuesta inmune robusta. ${ }^{100}$ Esto sugiere que la temperatura endógena humana es el principal obstáculo para su uso, como agente terapéutico eficaz.

\section{Esterasas mutantes}

Dado que la esterasa bacteriana es inestable a temperaturas fisiológicas, diversos grupos de investigación realizaron una serie de mutaciones encaminadas a mejorar la estabilidad de la proteína a diferentes temperaturas. Los mutantes, denominados T172R, G173Q y L196K mostraron una estabilidad significativa in vitro, a $37^{\circ} \mathrm{C}$. Cuando se evaluó, in vivo, el mutante T172R mostró una vida media de 78 minutos, mientras que los mutantes G173Q y L196K tuvieron una vida media de 75 y 403 minutos, respectivamente, a $37^{\circ} \mathrm{C}$. Con respecto a su actividad hidrolítica, el mutante G173Q no mostró ninguna alteración en su actividad catalítica; en cambio, el mutante T172R y el doble mutante T172R-G173Q mostraron un incremento (de tres veces) en la capacidad para hidrolizar a la cocaína. En cambio, el mutante L196K mostró un incremento (de ocho veces) en su eficiencia catalítica. ${ }^{101,102}$

De manera paralela, Gao et al., con el afán de aumentar la actividad hidrolítica de la BChE, generaron un mutante denominado AME-359. ${ }^{103}$ Esta enzima demostró una capacidad impresionante para hidrolizar a la cocaína en el plasma. ${ }^{104,105} \mathrm{Su}$ eficiencia catalítica se incrementó x100 veces más que la actividad catalítica mostrada por la BChE nativa humana y fue 450 veces más alta que la reportada para la CocE y la bCocE. ${ }^{66,98}$

Cuando se administró la AME-359 en una dosis de 0.5 $\mathrm{mg} / \mathrm{kg}$ disminuyó de manera más eficiente, en comparación con el tratamiento con $3 \mathrm{mg} / \mathrm{kg}$ de CocE, la toxicidad cardiovascular inducida por una sobredosis de cocaína. ${ }^{106}$

Recientemente se ha descrito la producción de mutantes de la BChE humana en plantas transgénicas (Nicotinia benthamiana). El primer mutante que se desarrolló utilizando esta aproximación fue un doble mutante de la BChE, el A328W/Y332A. Este mutante mostró un aumento significativo en su actividad hidrolítica contra la cocaína. ${ }^{107}$

Las propiedades catalíticas de este mutante (llamado variante 1) fueron subsecuentemente mejoradas al introducir mutaciones adicionales en diferentes sitios de la BHcE humana para crear las llamadas: variante 2 (F227A/ S287G/A328W/Y332A), variante 3 (A199S/S287G/A328W/ Y332G), variante 4 (A199S/F227A/S287G/A328W/Y332G) y 5 (F227A/S287G/A328W/Y332G), siendo la variante 4 de la BChE humana la más eficiente en hidrolizar a la cocaína. ${ }^{107,108}$

Recientemente, Hou et al. evaluaron la capacidad catalítica de dos mutantes de la BChE humana, el E14-3 y el E12-7, para hidrolizar a la cocaína-etileno, un producto toxico de la cocaína. In vitro, la enzima E12-7 mejoró hasta por 817 veces la eficiencia catalítica de la BChE humana; in vivo, la E12-7 fue capaz de hidrolizar eficientemente en ratas a la cocaína-etileno, a la cocaína y a la norcocaina. ${ }^{109}$

\section{Terapia génica}

Otros grupos de investigación desarrollaron y validaron otros protocolos de transferencia genómica, donde se transfirió el gen de la $\mathrm{CocH}$ humana a un huésped, por medio de un vector adenoviral, con el fin de generar altos y sostenidos 
(por algunos meses) niveles plasmáticos de la hidrolasa de cocaína. Para ello, el DNAc de la CocE humana fue incorporada dentro de un vector adenoviral tipo 5 con un promotor de citomegalovirus (hdAD), ${ }^{110}$ el cual podía traducir el gen de la CocE humana por algunos días o semanas, en ratas, generando notorias y sostenidas cantidades de la hidrolasa en el hígado, ${ }^{111}$ incrementando la eficiencia catalítica de la proteína traducida, hdAD-CocH, comparada con la BChE de rata, hasta por un factor de 50000 veces más. ${ }^{106}$

Otros estudios reportaron que la administración de dosis altas del vector, elevaron la actividad catalítica de la $\mathrm{CocH}$ hasta por 1000000 veces, sin reacciones secundarias aparentes. ${ }^{112-114}$ De hecho, Murthy et al. reportaron que la terapia de transferencia del vector hdAD-mCocH no generó efectos adversos secundarios sobre el funcionamiento del sistema colinérgico; los sujetos mostraron funciones cognitivas y motoras inalteradas. ${ }^{115}$

La administración del vector hdAD-CocH $(3 \mathrm{mg} / \mathrm{kg})$ a ratas o ratones disminuyó la vida media de la cocaína y atenuó los efectos cardiovasculares inducidos por diferentes dosis. ${ }^{111}$ Además, disminuyó dramáticamente el restablecimiento de la búsqueda de la droga en el modelo de auto-administración $(0.4 \mathrm{mg} / \mathrm{kg})$ hasta por seis meses, ${ }^{111}$ pero no alteró la conducta de ingestión de agua o alimento, tampoco modificó la auto-administración de anfetaminas $(0.05 \mathrm{mg} / \mathrm{kg})$ ni disminuyó la actividad locomotora. ${ }^{116}$

Esto sugiere que el vector hdAD-mCocH no alteró la eficiencia motora o la motivación relacionada a la búsqueda de la droga, al contrario, su efecto es específico para el reforzamiento producido por la cocaína. ${ }^{64}$

Recientemente se reportó la transferencia de la mutante de la CocH humana, AME359, a ratas, mediante el vector hdAD-hCocH. La administración de este vector disminuyó la concentración de cocaína en el plasma, previno la actividad locomotora inducida por ella, el restablecimiento de la conducta de búsqueda de la droga hasta por seis meses y disminuyó la letalidad después de una sobredosis, en ratas $(120 \mathrm{mg} / \mathrm{kg}){ }^{114}$

Otros estudios han reportado la transferencia de la $\mathrm{CocH}$ bacteriana mediante el uso de bacteriófagos. Los bacteriófagos son virus que tienen la capacidad de entrar al torrente sanguíneo y cruzar la barrera hemato-encefálica con facilidad; son capaces de tolerar una variedad de condiciones adversas como Ph extremos y tratamientos con nucleasas y enzimas proteolíticas, ${ }^{117}$ lo que hace al bacteriófago un buen medio a través del cual se puede transferir al Sistema Nervioso Central moléculas exógenas, que por su tamaño el sistema inmune o enzimático del huésped las pueden retirar rápidamente de la circulación, como la $\mathrm{CocH}$.

Howell et al. reportaron que la transferencia de la CocH bacteriana, mediante un bacteriófago, a Macacos Rhesus, eliminó a la cocaína del cerebro tres veces más rápido que cuando se administró de manera sistémica. Esta ruta de administración atenuó los efectos reforzantes de la co- caína ${ }^{118}$ y evitó el incremento en la presión sanguínea y en la frecuencia cardiaca después de la administración de una sobredosis. ${ }^{105}$

Rogers et al. lograron la expresión de la CocE humana usando la proteína III (pIII) y la proteína IX (pIX) de la cubierta de un bacteriófago. Ambas preparaciones, CocE-pIII $\mathrm{y}-\mathrm{pIX}$, fueron reproducibles y generaron una alta actividad catalítica. $^{15}$

Recientemente, Murthy et al. lograron transferir una BChE mutada a ratones. La transferencia mediante un vector viral elevó los niveles de la enzima 1000 veces, en comparación con los niveles normales, incrementando la capacidad catalítica de la enzima por meses, siendo capaz de eliminar a la cocaína en cuestión de segundos después de aparecer en el torrente sanguíneo. Adicionalmente, la BChE mutada fue capaz de atenuar la preferencia de lugar y disminuir la presión sanguínea y la letalidad inducida por una sobredosis $(80 \mathrm{mg} / \mathrm{kg}) \cdot{ }^{119}$

\section{Terapia dual}

Uno de los principales efectos de la administración de sobredosis de cocaína (100-120 mg/kg, ip.) es el daño permanente a los músculos y al hígado. ${ }^{112}$ Las terapias individuales como la administración de CocE humana $(0.3$ o $1 \mathrm{mg} / \mathrm{kg})$ o de anticuerpos monoclonales (10 o $20 \mathrm{mg} / \mathrm{kg}$ ), o la inmunización con un conjugado inmunogénico capaz de producir anticuerpos contra la cocaína, no han sido capaces de evitar estas alteraciones. Recientemente se ha reportado que el tratamiento con una combinación de estos agentes terapéuticos (enzima, $1 \mathrm{mg} / \mathrm{kg}$-anticuerpo, $8 \mathrm{mg} / \mathrm{kg}$, enzima, $1 \mathrm{mg} / \mathrm{kg}-100 \mu \mathrm{g}$ KLH-Norcocaína) proporcionó una completa protección al hígado y a los músculos. ${ }^{112,113}$ Además bloqueó completamente la estimulación locomotora inducida por $10 \mathrm{mg} / \mathrm{kg}$ de cocaína, ${ }^{21}$ lo cual sugiere que la combinación de diferentes terapias podría incrementar la protección contra las acciones psicoestimulantes de la cocaína y sustentan su uso en humanos como terapias de apoyo para lograr mantener la abstinencia. ${ }^{120}$

\section{DISCUSIÓN Y CONCLUSIÓN}

Como se mencionó anteriormente, a la fecha actual se carece de una terapia farmacológica eficaz en contra de los efectos inducidos por la cocaína, ${ }^{1,2}$ sobre todo en situaciones de intoxicación por sobredosis. Una opción terapéutica es el uso y validación de nuevas terapias alternativas. ${ }^{3,4}$

En un gran porcentaje, los adictos a la cocaína fallecen cuando consumen sobredosis, lo que provoca alteraciones de tipo cardiovascular, cerebro-vascular, convulsiones y/o la muerte. De tal forma, basados en la necesidad urgente de contar con una estrategia terapéutica alternativa, la validación del uso de enzimas (BChE, $\mathrm{COCH}$ y CoCe bacte- 
riana) capaces de disminuir significativamente los niveles de dosis, aun en rangos letales de cocaína, tanto en la sangre como en el cerebro,, 10 le proporcionaría a los servicios de emergencia una herramienta terapéutica única que les permitiría disminuir eficazmente los efectos letales de una sobredosis. ${ }^{121}$ Adicionalmente a su uso en situaciones de intoxicación por sobredosis, estudios en animales permiten sustentar el potencial terapéutico del uso de estas enzimas en humanos para inactivar rápidamente a la cocaína y desarrollar tratamientos para evitar las recaídas y mantener la abstinencia. ${ }^{122,123}$

Estudios clínicos de fase I han mostrado que la transferencia de la BChE humana pura o recombinante (TV-138) a sujetos sanos fue una terapia bien tolerada y segura. ${ }^{124} \mathrm{El}$ tratamiento con diferentes dosis (50, 100 y $300 \mathrm{mg}$ ) de la BChE-TV-138 facilitó la abstinencia en pacientes dependientes a la cocaína, disminuyó su uso y atenuó los efectos reforzantes subjetivos generados por la droga. ${ }^{125,126}$

Aunque estos estudios sugieren que una terapia basada en el uso de una BChE humana es segura y podría ser útil en mantener la abstinencia en sujetos dependientes; como sucede con otras terapias como la vacunación activa o pasiva, esta terapia tiene ciertas limitaciones: 1) su eficiencia depende de la permanencia de la enzima en el torrente sanguíneo, 2) es una terapia que sólo podrá evitar que la droga cruce la barrera hemato-encefálica de manera temporal, no por periodos prolongados de tiempo; por lo tanto, 3) su uso está restringido a ciertas poblaciones de sujetos, sobre todo aquellos que se encuentren en una situación de intoxicación por una sobredosis.

En este sentido, se requiere de estudios futuros en los que se evalúe la eficacia y la seguridad biológica del uso de esta terapia, junto con terapias farmacológicas, inmuno-farmacológicas o psicológicas.

Por otro lado, esta revisión bibliográfica tiene una serie de limitaciones: a) la búsqueda bibliográfica no se amplió a otros motores de búsqueda como Biological abstracts, Google Schoolar, Live Search Academic, etc., b) no se realizó el truncamiento de los descriptores utilizados, c) no se llevó a cabo la revisión de las referencias bibliográficas de los artículos incluidos en la revisión y d) el poco número de trabajos encaminados a describir el uso de esta estrategia terapéutica en humanos en situaciones de intoxicación por sobredosis de cocaína, limita las conclusiones.

Estos estudios, sugieren que el aumento en la actividad catalítica de las enzimas BChE y las hCE, podría ser una estrategia útil para desarrollar una terapia alternativa para el tratamiento de pacientes en condiciones de toxicidad por sobredosis de cocaína.

\section{Financiamiento}

Este trabajo recibió financiamiento de la Fundación Gonzalo Ríos Arronte, INP-2040.

\section{Conflicto de intereses}

Los autores declaran no tener ningún conflicto de interés.

\section{REFERENCIAS}

1. Fulco CE, Liverman CT, Earley LE editores. Development of medications for the treatment of opiate and cocaine addictions: Issues for the government and private sector, Washington, DC. National Academies Press; 1995.

2. Skolnick P. Biologic approaches to treat substance-use disorders. Trends Pharmacol Sci 2015;36(10):628-635.

3. Zalewska-Kaszubska J. Is immunotherapy an opportunity for effective treatment of drug addiction? Vaccine 2015;33(48):6545-6551.

4. Lockridge O. Review of human butyrylcholinesterase structure, function, genetic variants, history of use in the clinic, and potential therapeutic uses. Pharmacol Ther 2015; 148(1):34-46.

5. Shorter D, Kosten TR. Novel pharmacotherapeutic treatments for cocaine addiction. BMC Med 2011;9(1):119.

6. Shorter D, Domingo CB, Kosten TR. Emerging drugs for the treatment of cocaine use disorder: a review of neurobiological targets and pharmacotherapy. Expert Opin Emerg Drugs 2015;20(1):15-29.

7. Orson FM, Wang R, Brimijoin S, Kinsey BM et al. The future potential for cocaine vaccines. Expert Opin Biol Ther 2014;14(9):1271-1283.

8. Brimijoin S, Shen X, Orson F, Kosten T. Prospects, promise and problems on the road to effective vaccines and related therapies for substance abuse. Expert Rev Vaccines 2013;12(3):323-332.

9. Brimijoin S. Interception of cocaine by enzyme or antibody delivered with viral gene transfer: a novel strategy for preventing relapse in recovering drug users. CNS Neurol Disord Drug Targets 2011;10(8):880-891.

10. Schindler CW, Goldberg SR. Accelerating cocaine metabolism as an approach to the treatment of cocaine abuse and toxicity. Future Med Chem 2012;4(2):163-175.

11. Zheng F, Zhan CG. Rational design of an enzyme mutant for anti-cocaine therapeutics. J Comput Aided Mol Des 2008;22(9):661-671.

12. Martins SS, Sampson L, Cerdá M, Galea S. Worldwide prevalence and trends in unintentional drug overdose: A systematic review of the literature. Am J Public Health 2015;105(11):e29-e49.

13. Bosron WF, Hurley TD. Lessons from a bacterial cocaine esterase. Nat Struct Biol 2002;9(1):4-5.

14. De Prada P, Winger G, Landry DW. Application of artificial enzymes to the problem of cocaine. Ann N Y Acad Sci 2000;909(1):159-169.

15. Rogers CJ, Eubanks LM, Dickerson TJ, Janda KD. Unexpected acetylcholinesterase activity of cocaine esterases. J Am Chem Soc 2006;128(48):15364-15365.

16. Turner JM, Larsen NA, Basran A, Barbas CF et al. Biochemical characterization and structural analysis of a highly proficient cocaine esterase. Biochemistry 2002;41(41):12297-12307.

17. Boeck AT, Schopfer LM, Lockridge O. DNA sequence of butyrylcholinesterase from the rat: expression of the protein and characterization of the properties of rat butyrylcholinesterase. Biochem Pharmacol 2002;63(12):2101-2110.

18. Gatley SJ. Activities of the enantiomers of cocaine and some related compounds as substrates and inhibitors of plasma butyrylcholinesterase. Biochem Pharmacol 1991;41(8):1249-1254.

19. Jbilo O, Bartels CF, Chatonnet A, Toutant JP et al. Tissue distribution of human acetylcholinesterase and butyrylcholinesterase messenger RNA. Toxicon 1994;32(11):1445-1457.

20. Gatley SJ, MacGregor RR, Fowler JS, Wolf AP et al. Rapid stereoselective hydrolysis of $(+)$-cocaine in baboon plasma prevents its uptake in the brain: implications for behavioral studies. J Neurochem 1990;54(2):720-723.

21. Loewenstein-Lichtenstein Y, Glick D, Gluzman N, Sternfeld M et al. Overlapping drug interaction sites of human butyrylcholi- 
nesterase dissected by site-directed mutagenesis. Mol Pharmacol 1996;50(6):1423-1431.

22. Zhan CG, Zheng F, Landry DW. Fundamental reaction mechanism for cocaine hydrolysis in human butyrylcholinesterase. J Am Chem Soc 2003;125(9):2462-2474.

23. Zhan CG, Deng SX, Skiba JG, Hayes BA et al. First-principle studies of intermolecular and intramolecular catalysis of protonated cocaine. J Comput Chem 2005;26(10):980-986.

24. Gorelick DA. Enhancing cocaine metabolism with butyrylcholinesterase as a treatment strategy. Drug Alcohol Depend 1997;48(3):159-165.

25. Lynch TJ, Mattes CE, Singh A, Bradley RM et al. Cocaine detoxification by human plasma butyrylcholinesterase. Toxicol Appl Pharmacol 1997;145(2):363-371.

26. Mattes CE, Lynch TJ, Singh A, Bradley RM et al. Therapeutic use of butyrylcholinesterase for cocaine intoxication. Toxicol Appl Pharmacol 1997; 45(2):372-380.

27. Morell V. Enzyme may blunt cocaine's action. Science 1993;259(5103):1828.

28. Schwarz M, Glick D, Loewenstein Y, Soreq H. Engineering of human cholinesterases explains and predicts diverse consequences of administration of various drugs and poisons. Pharmacol Ther 1995;67(2):283-322.

29. Erzouki HK, Baum I, Goldberg SR, Schindler CW. Comparison of the effects of cocaine and its metabolites on cardiovascular function in anesthetized rats. J Cardiovasc Pharmacol 1993;22(4):557-563.

30. Madden JA, Powers RH. Effect of cocaine and cocaine metabolites on cerebral arteries in vitro. Life Sci 1990;47(13):1109-1114.

31. Konkol RJ, Erickson BA, Doerr JK, Hoffman RG et al. Seizures induced by the cocaine metabolite benzoylecgonine in rats. Epilepsia 1992;33(3):420-427.

32. Ndikum-Moffor FM, Schoeb TR, Roberts SM. Liver toxicity from norcocaine nitroxide, an $\mathrm{N}$-oxidative metabolite of cocaine. J Pharmacol Exp Ther 1998;284(1):413-419.

33. Rauckman EJ, Rosen GM, Cavagnaro J. Norcocaine nitroxide. A potential hepatotoxic metabolite of cocaine. Mol Pharmacol 1982;21(2):458463.

34. Carmona GN, Schindler CW, Greig NH, Holloway HW et al. Intravenous butyrylcholinesterase administration and plasma and brain levels of cocaine and metabolites in rats. Eur J Pharmacol 2005;517(3):186190.

35. Zhan CG, Gao D. Catalytic mechanism and energy barriers for butyrylcholinesterase-catalyzed hydrolysis of cocaine. Biophys J. 2005; 89(6):3863-3872.

36. Zheng F, Zhan CG. Recent progress in protein drug design and discovery with a focus on novel approaches to the development of anti-cocaine medications. Future Med Chem. 2009;1(3):515-528.

37. Brimijoin S, Gao Y. Cocaine hydrolase gene therapy for cocaine abuse. Future Med Chem 2012;4(2):151-162.

38. Dimov D, Kanev K, Dimova I. Correlation between butyrylcholinesterase variants and sensitivity to soman toxicity. Acta Biochim Pol 2012;59(2):313-316.

39. Negrão AB, Pereira AC, Guindalini C, Santos HC, Messas GP, Laranjeira $R$, Vallada $H$. Butyrylcholinesterase genetic variants: association with cocaine dependence and related phenotypes. PLoS One 2013;8(11):e80505.

40. Devenyi P. Cocaine complications and pseudocholinesterase. Ann Intern Med 1989;110(2):167-168.

41. Hoffman RS, Henry GC, Howland MA, Weisman RS, Weil L, Goldfrank LR. Association between life-threatening cocaine toxicity and plasma cholinesterase activity. Ann Emerg Med 1992;21(3):247-253.

42. Hoffman RS, Henry GC, Wax PM, Weisman RS, Howland MA, Goldfrank LR. Decreased plasma cholinesterase activity enhances cocaine toxicity in mice. J Pharmacol Exp Ther 1992;263(2):698-702.

43. Liddell J, Lehmann H, Silk E. A 'silent' pseudo-cholinesterase gene. Nature 1962;193(2):561-562.
44. Manoharan I, Boopathy R, Darvesh S, Lockridge O. A medical health report onindividuals with silent butyrylcholinesterase in the Vysya community of India. Clin Chim Acta. 2007; 378(1):128-135.

45. Jatlow P, Barash PG, Van Dyke C, Radding J, Byck R. Cocaine and succinylcholine sensitivity: a new caution. Anesth Analg 1979;58(3):235238.

46. Stewart DJ, Inaba T, Lucassen M, Kalow W. Cocaine metabolism: cocaine and norcocaine hydrolysis by liver and serum esterases. Clin Pharmacol Ther 1979;25(4):464-468.

47. Goedde HW, Altland K. Evidence for different "silent genes" in the human serum pseudocholinesterase polymorphism. Ann N Y Acad Sci 1968;151(1):540-544.

48. Klose R, Gutensohn G. Treatment of alkyl phosphate poisoning with purified serum cholinesterase. Prakt Anaesth 1976;11(1):1-7.

49. Duysen EG, Li B, Lockridge O. The butyrylcholinesterase knockout mouse a research tool in the study of drug sensitivity, bio-distribution, obesity and Alzheimer's disease. Expert Opin Drug Metab Toxicol 2009;5(5):523-528.

50. Duysen EG, Lockridge O. Prolonged toxic effects after cocaine challenge in butyrylcholinesterase/plasma carboxylesterase double knockout mice: a model for butyrylcholinesterase-deficient humans. Drug Metab Dispos 2011;39(8):1321-1323.

51. Ralph EC, Xiang L, Cashman JR, Zhang J. His-tag truncated butyrylcholinesterase as a useful construct for in vitro characterization of wild-type and variant butyrylcholinesterases. Protein Expr Purif 2011;80(1):22-27.

52. Carmona GN, Jufer RA, Goldberg SR, Gorelick DA et al. Butyrylcholinesterase accelerates cocaine metabolism: in vitro and in vivo effects in nonhuman primates and humans. Drug Metab Dispos 2000;28(3):367-371.

53. Mattes C, Bradley R, Slaughter E, Browne S. Cocaine and butyrylcholinesterase (BChE): determination of enzymatic parameters. Life Sci 1996;58(13):PL257-261.

54. Browne SP, Slaughter EA, Couch RA, Rudnic EM et al. The influence of plasma butyrylcholinesterase concentration on the in vitro hydrolysis of cocaine in human plasma. Biopharm Drug Dispos 1998;19(5):309-314.

55. Carmona GN, Baum I, Schindler CW, Goldberg SR et al. Plasma butyrylcholinesterase activity and cocaine half-life differ significantly in rhesus and squirrel monkeys. Life Sci 1996;59(11):939-943.

56. Carmona GN, Schindler CW, Shoaib M, Jufer R et al. Attenuation of cocaine-induced locomotor activity by butyrylcholinesterase. Exp Clin Psychopharmacol 1998;6(3):274-279.

57. Brimijoin S, Shen ML, Sun H. Radiometric solvent-partitioning assay for screening cocaine hydrolases and measuring cocaine levels in mi1ligram tissue samples. Anal Biochem 2002;309(2):200-205.

58. Koetzner L, Woods JH. Characterization of butyrylcholinesterase antagonism of cocaine-induced hyperactivity. Drug Metab Dispos 2002;30(6):716-723.

59. Koetzner L, Woods JH. Characterization of equine butyrylcholinesterase disposition in the mouse. Drug Metab Dispos 2002;30(6):724-730.

60. Sáez-Valero J, de Gracia JA, Lockridge O. Intraperitoneal administration of $340 \mathrm{kDa}$ human plasma butyrylcholinesterase increases the level of the enzyme in the cerebrospinal fluid of rats. Neurosci Lett 2005;383(1):93-98. Epub 2005 Apr 12.

61. Brimijoin S, Gao Y, Anker JJ, Gliddon LA et al. A cocaine hydrolase engineered from human butyrylcholinesterase selectively blocks cocaine toxicity and reinstatement of drug seeking in rats. Neuropsychopharmacology 2008;33(11):2715-2725.

62. Carroll ME, Gao Y, Brimijoin S, Anker JJ. Effects of cocaine hydrolase on cocaine self-administration under a PR schedule and during extended access (escalation) in rats. Psychopharmacology (Berl) 2011;213(4):817-829.

63. Schindler CW, Justinova Z, Lafleur D, Woods D et al. Modification of pharmacokinetic and abuse-related effects of cocaine by human-derived cocaine hydrolase in monkeys. Addict Biol 2013;18(1):30-39. 
64. Zlebnik NE, Brimijoin S, Gao Y, Saykao AT et al. Long-term reduction of cocaine self-administration in rats treated with adenoviral vector-delivered cocaine hydrolase: Evidence for enzymatic activity. Neuropsychopharmacology 2014;39(6):1538-1546.

65. Collins GT, Carey KA, Narasimhan D, Nichols J et al. Amelioration of the cardiovascular effects of cocaine in rhesus monkeys by a long-acting mutant form of cocaine esterase. Neuropsychopharmacology 2011;36(5):1047-1059.

66. Collins GT, Zaks ME, Cunningham AR, St Clair C et al. Effects of a long-acting mutant bacterial cocaine esterase on acute cocaine toxicity in rats. Drug Alcohol Depend 2011;118(2):158-165.

67. Lockridge O, Schopfer LM, Winger G, Woods JH. Large scale purification of butyrylcholinesterase from human plasma suitable for injection into monkeys; a potential new therapeutic for protection against cocaine and nerve agent toxicity. J Med Chem Biol Radiol Def $2005 \mathrm{Jul}$ 1;3:nihms5095.

68. Gao Y, LaFleur D, Shah R, Zhao Q et al. An albumin-butyrylcholinesterase for cocaine toxicity and addiction: catalytic and pharmacokinetic properties. Chem Biol Interact 2008;175(1):83-87.

69. Berkman CE, Underiner GE, Cashman JR. Stereoselective inhibition of human butyrylcholinesterase by phosphonothiolate analogs of (+)and (-)-cocaine. Biochem Pharmacol 1997;54(11):1261-1266.

70. Visalli T, Turkall R, Abdel-Rahman MS. Plasma butyrylcholinesterase activity protects against cocaine hepatotoxicity in female mice. Toxicol Mech Methods 2005;15(6):383-389.

71. Fang L, Hou S, Xue L, Zheng F et al. Amino-acid mutations to extend the biological half-life of a therapeutically valuable mutant of human butyrylcholinesterase. Chem Biol Interact 2014;214(1):18-25.

72. Hamza A, Cho H, Tai HH, Zhan CG. Molecular dynamics simulation of cocaine binding with human butyrylcholinesterase and its mutants. J Phys Chem B 2005;109(10):4776-4782.

73. Pan $\mathrm{Y}$, Gao D, Yang W, Cho $\mathrm{H}$ et al. Free energy perturbation (FEP) simulation on the transition states of cocaine hydrolysis catalyzed by human butyrylcholinesterase and its mutants. J Am Chem Soc 2007;129(44):13537-13543.

74. Duysen EG, Bartels CF, Lockridge O. Wild-type and A328W mutant human butyrylcholinesterase tetramers expressed in Chinese hamster ovary cells have a 16-hour half-life in the circulation and protect mice from cocaine toxicity. J Pharmacol Exp Ther 2002;302(2):751-758.

75. Xie W, Altamirano CV, Bartels CF, Speirs RJ et al. An improved cocaine hydrolase: the $\mathrm{A} 328 \mathrm{Y}$ mutant of human butyrylcholinesterase is 4-fold more efficient. Mol Pharmacol 1999;55(1):83-91.

76. Zheng F, Yang W, Ko MC, Liu J et al. Most efficient cocaine hydrolase designed by virtual screening of transition states. J Am Chem Soc 2008;130(6):12148-12155.

77. Zheng F, Zhan CG. Structure-and-mechanism-based design and discovery of therapeutics for cocaine overdose and addiction. Org Biomol Chem 2008;6(5):836-843.

78. Zheng $\mathrm{F}$, Yang $\mathrm{W}$, Xue L, Hou $\mathrm{S}$ et al. Design of high-activity mutants of human butyrylcholinesterase against (-)-cocaine: structural and energetic factors affecting the catalytic efficiency. Biochemistry 2010;49(42):9113-9119.

79. Zheng F, Xue L, Hou S, Liu J et al. A highly efficient cocaine-detoxifying enzyme obtained by computational design. Nat Commun 2014;5:3457.

80. Zheng F, Zhan M, Huang X, Abdul Hameed MD et al. Modeling in vitro inhibition of butyrylcholinesterase using molecular docking, multi-linear regression and artificial neural network approaches. Bioorg Med Chem 2014;22(1):538-549.

81. Huang X, Pan Y, Zheng F, Zhan CG. Reaction pathway and free energy profile for prechemical reaction step of human butyrylcholinesterase-catalyzed hydrolysis of (-)-cocaine by combined targeted molecular dynamics and potential of mean force simulations. J Phys Chem B 2010;114(42):13545-13554.

82. Huang $X$, Zheng F, Zhan CG. Human butyrylcholinesterase-cocaine binding pathway and free energy profiles by molecular dynamics and potential of mean force simulations. J Phys Chem B 2011;115(38):1125411260.

83. Mikami LR, Wieseler S, Souza RL, Schopfer LM et al. Five new naturally occurring mutations of the BCHE gene and frequencies of 12 butyrylcholinesterase alleles in a Brazilian population. Pharmacogenet Genomics 2008;18(3):213-218.

84. Hou S, Xue L, Yang W, Fang L et al. Substrate selectivity of high-activity mutants of human butyrylcholinesterase. Org Biomol Chem 2013;11(43):7477-7485.

85. Yang W, Pan Y, Fang L, Gao D et al. Free energy perturbation simulation on transition states and high-activity mutants of human butyrylcholinesterase for (-)-cocaine hydrolysis. J Phys Chem B 2010;114(33):10889-10896.

86. Yang $\mathrm{W}$, Xue L, Fang $\mathrm{L}$, Chen $\mathrm{X}$ et al. Characterization of a high-activity mutant of human butyrylcholinesterase against (-)-cocaine. Chem Biol Interact 2010;187(1-3):148-152.

87. Gao $Y$, Brimijoin S. Lasting reduction of cocaine action in neostriatum-a hydrolase gene therapy approach. J Pharmacol Exp Ther 2009;330(2):449-457.

88. Zhan M, Hou S, Zhan CG, Zheng F. Kinetic characterization of high-activity mutants of human butyrylcholinesterase for the cocaine metabolite norcocaine. Biochem J 2014;457(1):197-206.

89. Gao Y, Brimijoin S. An engineered cocaine hydrolase blunts and reverses cardiovascular responses to cocaine in rats. J Pharmacol Exp Ther 2004;310(3):1046-1052.

90. Sun H, Pang YP, Lockridge O, Brimijoin S. Re-engineering butyrylcholinesterase as a cocaine hydrolase. Mol Pharmacol 2002;62(2):220224.

91. Sun H, Shen ML, Pang YP, Lockridge O et al. Cocaine metabolism accelerated by a re-engineered human butyrylcholinesterase. J Pharmacol Exp Ther 2002;302(2):710-716.

92. Xue L, Ko MC, Tong $M$, Yang $W$ et al. Design, preparation, and characterization of high-activity mutants of human butyrylcholinesterase specific for detoxification of cocaine. Mol Pharmacol 2011;79(2):290297.

93. Xue L, Hou S, Tong M, Fang L et al. Preparation and in vivo characterization of a cocaine hydrolase engineered fromhuman butyrylcholinesterase for metabolizing cocaine. Biochem J 2013;453(3):447-454.

94. Ko MC, Narasimhan D, Berlin AA, Lukacs NW et al. Effects of cocaine esterase following its repeated administration with cocaine in mice. Drug Alcohol Depend 2009;101(3):202-209.

95. Narasimhan D, Woods JH, Sunahara RK. Bacterial cocaine esterase: a protein-based therapy for cocaine overdose and addiction. Future Med Chem 2012;4(2):137-150.

96. Ascenzi P, Clementi E, Polticelli F. The Rhodococcus sp. cocaine esterase: a bacterial candidate for novel pharmacokinetic-based therapies for cocaine abuse. IUBMB Life 2003;55(7):397-402.

97. Cooper ZD, Narasimhan D, Sunahara RK, Mierzejewski P et al. Rapid and robust protection against cocaine-induced lethality in rats by the bacterial cocaine esterase. Mol Pharmacol 2006;70(6):1885-1891.

98. Collins GT, Brim RL, Narasimhan D, Ko MC et al. Cocaine esterase prevents cocaine-induced toxicity and the ongoing intravenous self-administration of cocaine in rats. J Pharmacol Exp Ther 2009;331(2):445-455.

99. Brim RL, Noon KR, Collins GT, Stein A et al. The fate of bacterial cocaine esterase (CocE): an in vivo study of CocE-mediated cocaine hydrolysis, CocE pharmacokinetics, and CocE elimination. J Pharmacol Exp Ther 2012;340(1):83-95.

100. Ko MC, Bowen LD, Narasimhan D, Berlin AA et al. Cocaine esterase: interactions with cocaine and immune responses in mice. J Pharmacol Exp Ther 2007;320(2):926-933.

101. Fang L, Chow KM, Hou S, Xue L et al. Rational design, preparation, and characterization of a therapeutic enzyme mutant with improved stability and function for cocaine detoxification. ACS Chem Biol 2014;9(8):1764-1772. 
102. Narasimhan D, Nance MR, Gao D, Ko MC et al. Structural analysis of thermostabilizing mutations of cocaine esterase. Protein Eng Des Sel 2010;23(7):537-547.

103. Gao Y, Brimijoin S. Visualizing viral transduction of a cocaine-hydrolyzing, human butyrylcholinesterase in rats. Chem Biol Interact 2005;157-158(1):97-103.

104. Collins GT, Narasimhan D, Cunningham AR, Zaks ME et al. Long-lasting effects of a PEGylated mutant cocaine esterase (CocE) on the reinforcing and discriminative stimulus effects of cocaine in rats. Neuropsychopharmacology 2012;37(5):1092-1103.

105. Collins GT, Brim RL, Noon KR, Narasimhan D et al. Repeated administration of a mutant cocaine esterase: effects on plasma cocaine levels, cocaine-induced cardiovascular activity, and immune responses in rhesus monkeys. J Pharmacol Exp Ther 2012;342(1):205-213.

106. Gao Y, Atanasova E, Sui N, Pancook JD et al. Gene transfer of cocaine hydrolase suppresses cardiovascular responses to cocaine in rats. Mol Pharmacol 2005;67(1):204-211.

107. Larrimore KE, Barcus M, Kannan L, Gao Y et al. Plants as a source of butyrylcholinesterase variants designed for enhanced cocaine hydrolase activity. Chem Biol Interact 2013;203(1):217-220.

108. Chen X, Huang X, Geng $L$, Xue $L$ et al. Kinetic characterization of a cocaine hydrolase engineered from mouse butyrylcholinesterase. Biochem J 2015;466(2):243-251.

109. Hou S, Zhan M, Zheng X, Zhan CG et al. Kinetic characterization of human butyrylcholinesterase mutants for the hydrolysis of cocaethylene. Biochem J 2014;460(3):447-457.

110. Chilukuri N, Duysen EG, Parikh K, Sun W et al. Adenovirus-mediated gene transfer of human butyrylcholinesterase results in persistent high-level transgene expression in vivo. Chem Biol Interact 2008;175(13):327-331.

111. Gao Y, Brimijoin S. Viral transduction of cocaine hydrolase in brain reward centers. Cell Mol Neurobiol 2006;26(4):357-363.

112. Brimijoin S, Orson F, Kosten TR, Kinsey B et al. Anti-cocaine antibody and butyrylcholinesterase-derived cocaine hydrolase exert cooperative effects on cocaine pharmacokinetics and cocaine-induced locomotor activity in mice. Chem Biol Interact 2013;203(1):212-216.

113. Gao Y, Geng L, Orson F, Kinsey B et al. Effects of anti-cocaine vaccine and viral gene transfer of cocaine hydrolase in mice on cocaine toxicity including motor strength and liver damage. Chem Biol Interact 2013;203(1):208-211.

114. Geng L, Gao Y, Chen X, Hou S et al. Gene transfer of mutant mouse cholinesterase provides high lifetime expression and reduced cocaine responses with no evident toxicity. PLoS One 2013;8(6):e67446.
115. Murthy V, Gao Y, Geng L, Lebrasseur N et al. Preclinical studies on neurobehavioral and neuromuscular effects of cocaine hydrolase gene therapy in mice. J Mol Neurosci 2014;53(3):409-416.

116. Anker JJ, Brimijoin S, Gao Y, Geng L et al. Cocaine hydrolase encoded in viral vector blocks the reinstatement of cocaine seeking in rats for 6 months. Biol Psychiatry 2012;71(8):700-705.

117. Dickerson TJ, Kaufmann GF, Janda KD. Bacteriophage-mediated protein delivery into the central nervous system and its application in immunopharmacotherapy. Expert Opin Biol Ther 2005;5(6):773-781.

118. Howell LL, Nye JA, Stehouwer JS, Voll RJ et al. A thermostable bacterial cocaine esterase rapidly eliminates cocaine from brain in nonhuman primates. Transl Psychiatry 2014;4:e407.

119. Murthy V, Geng L, Gao Y, Zhang B et al. Reward and toxicity of cocaine metabolites generated by cocaine hydrolase. Cell Mol Neurobiol 2015;35(6): 819-826.

120. Carroll ME, Zlebnik NE, Anker JJ, Kosten TR et al. Combined cocaine hydrolase gene transfer and anti-cocaine vaccine synergistically block cocaine-induced locomotion. PLoS One 2012;7(8):e43536.

121. Connors NJ, Hoffman RS. Experimental treatments for cocaine toxicity: a difficult transition to the bedside. J Pharmacol Exp Ther 2013;347(2):251-257.

122. Askalsky P, Kalapatapu RK, Foltin RW, Comer SD. Butyrylcholinesterase levels and subjective effects of smoked cocaine in healthy cocaine users. Am J Drug Alcohol Abuse 2015;41(2):161-165.

123. Lockridge O. Review of human butyrylcholinesterase structure, function, genetic variants, history of use in the clinic, and potential therapeutic uses. Pharmacol Ther 2015;148(1):34-46.

124. Murthy V, Gao Y, Geng L, LeBrasseur NK et al. Physiologic and metabolic safety of butyrylcholinesterase gene therapy in mice. Vaccine 2014;32(33):4155-4162.

125. Cohen-Barak O, Wildeman J, van de Wetering J, Hettinga J et al. Safety, pharmacokinetics, and pharmacodynamics of TV-1380, a novel mutated butyrylcholinesterase treatment for cocaine addiction, after single and multiple intramuscular injections in healthy subjects. J Clin Pharmacol 2015;55(5):573-583.

126. Shram MJ, Cohen-Barak O, Chakraborty B, Bassan M et al. Assessment of pharmacokinetic and pharmacodynamic interactions between albumin-fused mutated butyrylcholinesterase and intravenously administered cocaine in recreational cocaine users. J Clin Psychopharmacol 2015;35(4):396-405. 